\title{
Virtual Simulation of Petrochemical Direct Operation Based on Self-Built Scenes
}

\author{
Shujun Zhang ${ }^{1}$ Weijia Meng ${ }^{2}$ Qiannan Wan ${ }^{3}$ Yunxia Feng ${ }^{4}$ \\ \{lindazsj@163.com ${ }^{1}, 386345026 @ q q . c^{2}$, 815955181@qq.com ${ }^{3}$, cloudy_feng@163.com $\left.{ }^{4}\right\}$ \\ College of Information Science and Technology,
}

Qingdao University of Science and Technology, Qingdao 266061

\begin{abstract}
To overcome the deficiencies of traditional training method based on texts and images, a virtual simulation method of petrochemical direct operation is proposed based on self-built scenes by means of virtual reality and simulation, 3D visualization, Internet technology and other advanced technology. According to the characteristics of direct operation such as strong systematicness, complex scenes, high risk and multi-role cooperation, this paper presents a new 3D modeling method based on large scale topographical map and chemical process to model the global and detailed information of petrochemical plants. Distributed interactive simulation technique is adopted for the communication and interaction between different trainees. We use event-based approach to training (EBAT) method and analyze elements of direction operation and logical relationship between different tasks to construct selfbuilt scenes and self-edited courses, so simulation training courses can be expanded indefinitely. Experimental results show the validity of the proposed method.
\end{abstract}

Keywords: direct operation; self-built scenes; virtual reality; distributed interactive simulation

\section{Introduction}

Petrochemical direct operation is a series of activities performed by workers at the scene in production process. Different from other operations, direct operation has many characteristics including high-risk, real-time response, step complexity, irreversibility, multi-role interaction, etc. Statistics of enterprise accidents in recent years show that the proportion of accidents in direct operation is as high as $80 \%$, and the causes of accidents mostly include two aspects, one is that the security awareness of the operators is weak and the other is that risk analysis is not comprehensive. Therefore, it is necessary to make safety training. The traditional training methods are mainly based on two-dimensional text and images, but the entire process of direct operation is a complex and dynamic process which is difficult to be expressed just through text or image, therefore this training method is ineffective.

With the development of virtual reality technology, virtual simulation training has attracted extensive attention of scientific research institutions, and the number of virtual reality-based systems is increasing. Automotive product design oriented virtual reality service platform [1] developed by Zhang Linxuan et al and energy station interactive display system [2] developed by He Gaoqi et al, both of them emphasized 3D visualization of the system and associated less with human-computer interaction; CNG simulation training system based on X3D [3] developed by Wu Dandan et al of Southwest Petroleum University explored the operation simulation and process flow simulation of $\mathrm{CNG}$ fueling station, but it could not achieve multi-people cooperation; Virtual Simulation System of Cutter Suction Dredger Based on Unity3D [4] developed by Yao Pengfei et al of Hohai University realized the information display, construction display and operation training, but it's also impossible to realize multi-people cooperation; Virtual simulation training system of small-scale workover based on multi-user collaboration [5] developed by Yue Yanyan et al of Northeast Petroleum University tested multi-people cooperation. It shared action, text chat and other information, but it could only be trained on the existing virtual simulation course, namely, it was not extendable. Most of the above simulation training systems are aimed at specific situations and single training content, which can't meet with requirements of direct operation.

In order to set up simulation training environment, Oser, R. L. at al presented event-based MOBIMEDIA 2018, June 21-22, Qingdao, People's Republic of 6 . People research on EBAT in China are Wang Jian and Wang 
and training conceptual model based on CMMS (Conceptual Model of the Mission Space) and a formal description method were raised on the basis of analyzing modeling elements of team training [8] in 2011. A general framework of scenario generation and scheduling based on EBAT [9] was proposed in 2012.

Based on EBAT principle, a virtual simulation technique of petrochemical direct operation based on self-built scenes are proposed in this paper. In our system, EBAT is supplemented by self-built scenes and self-edited courses. The selective information synchronization for multiple people in the same scene ensures the effective execution of system logic while conducting multiperson training, by the means of distributed interactive simulation technology and selective interactive communication technology. Trainers get a score evaluation and feedback automatically according to interaction with simulation system after the training. Our work not only ensures the long-term effectiveness of the system, but also fills the vacancy of simulation training in petrochemical direct operation.

\section{Simulation of Direct Operation}

Virtual reality has three basic characteristics: immersion, imagination and interaction, and immersion requires that users feel the same in virtual environment as in real environment, interaction requires that users operate reasonably and effectively in virtual environment and can get real-time feedback in real environment.

The whole design flow of the proposed simulation technique based on EBAT is shown in Figure 1:

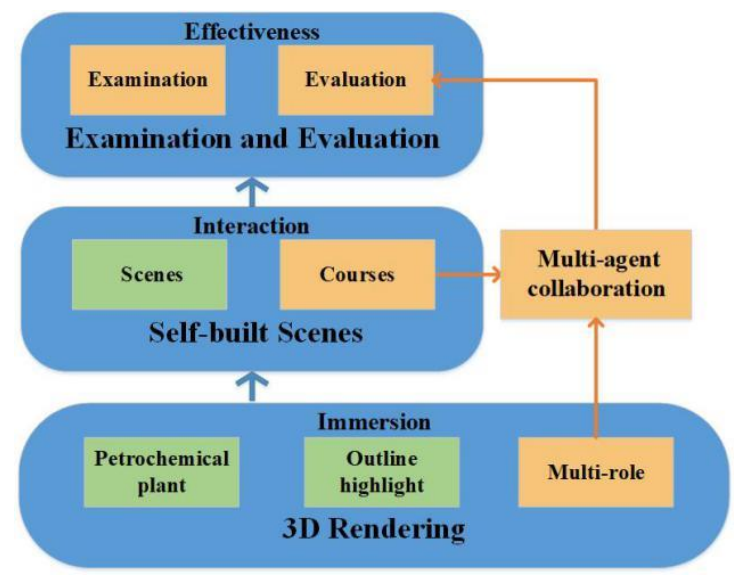

Fig. 1. Design flow of the proposed simulation technique based on EBAT

In Figure 1, we divide all the components into three layers, the first layer is the 3D rendering layer which includes petrochemical plant 3D modeling, multi-role characters and outline highlight of the interactive objects. The second layer is the self-built scene layer to edit training scenes and courses based on EBAT. The third layer is the examination and evaluation layer, which is responsible for the feedback and grading of the trainers" performance. Multiagent collaboration occurs through multi-role modeling, event editing, assessment and evaluation.

\subsection{D Rendering}

Three-dimensional modeling of petrochemical plant based on chemical process flow. Fidelity of virtual environment directly affects the usability of the virtual simulation. A new 3D modeling method based on large scale topographical map is applied to modeling petrochemical plant quickly and accurately due to the complexity of scenes of petrochemical plant. Because large scale topographic maps contain the basic geospatial information of the petrochemical plant, accurate $3 \mathrm{D}$ reconstruction can be done according to this method with supplying other forms of data. However, complicated petrochemical plant has a large amount of pipelines, large scale topographic maps can't cover the pipeline information accurately.

In the chemical industry, raw materials is processed on a large scale. It changes not only in its state and physical properties, but also in its chemical properties. It eventually becomes a product that meet the needs. The process is called chemical process, which involves many steps. Raw materials become products after passing through several equipment in sequence and processing in various ways in all steps called process flow [10]. The specific layout of the pipeline can be known by obtaining the trend of the raw material in the pipeline and the function 
of each equipment. So, the pipeline can be modeled and placed accurately.

In summary, equipment can be modeled and placed according to large scale topographic maps, photo and other information of petrochemical plant. Pipelines can be modeled and placed by chemical process flow. Equipment and pipelines can be restored truly by the two methods. Three-dimensional scene of petrochemical plant by this modeling method is shown in Figure 2.

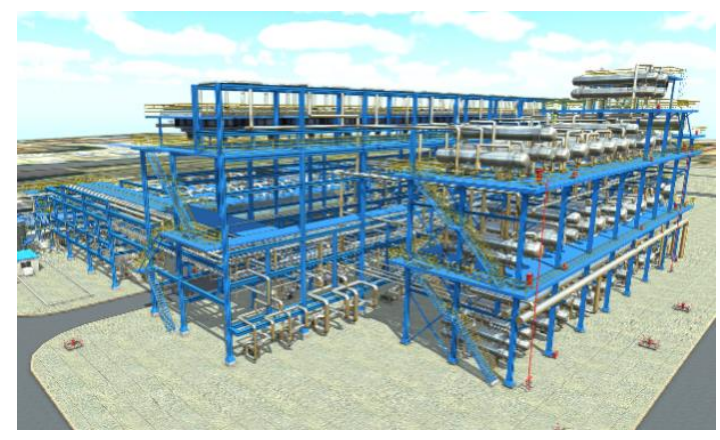

Fig. 2. Three-dimensional modeling of petrochemical plant

Multi-role modeling. The implementation of a direct operation requires the participation of seven types of roles. In order to simulate the characteristics of multi-role participation in direct operation, we model different kinds of operators according to the scale of real people.

Outline highlighting based on rim light. In order to recognize 3D model clearly in the virtual simulation environment, outline highlighting of the model is required. In Unity3D, color variation of the model is controlled by Shader of material. Outline highlighting of the model is accomplished by rim light of Shader, and specific algorithms are as follows:

$$
\begin{aligned}
& \mathrm{R}(\mathrm{v}, \mathrm{n})=1-\vec{v} \cdot \vec{n} \\
& \mathrm{E}=R^{m}
\end{aligned}
$$

In Formula (1), the $\mathrm{v}$ is the current viewing angle, the $\mathrm{n}$ is the normal of target, when the angle between the two is ninety degrees, the $\mathrm{R}$ gets the maximum, that is, the light up to 90 degrees from current viewing angle is the strongest, and the rim light is the strongest. In Formula (2), the value of $\mathrm{R}$ is magnified in an exponential form. Implementation mode is as follow in Unity3D:

half rim $=1.0-$ saturate $(\operatorname{dot}$ (normalize(IN.viewDir), IN.worldNormal));

o.Emission = _RimColor.rgb * POW (rim,_RimPower);

The IN.viewDir is the current view vector, The IN.worldNormal is the normal of the model, and the dot is the dot product of the view vector and the normal of the model. The effect of outline highlighting by this method is shown in Figure 3.

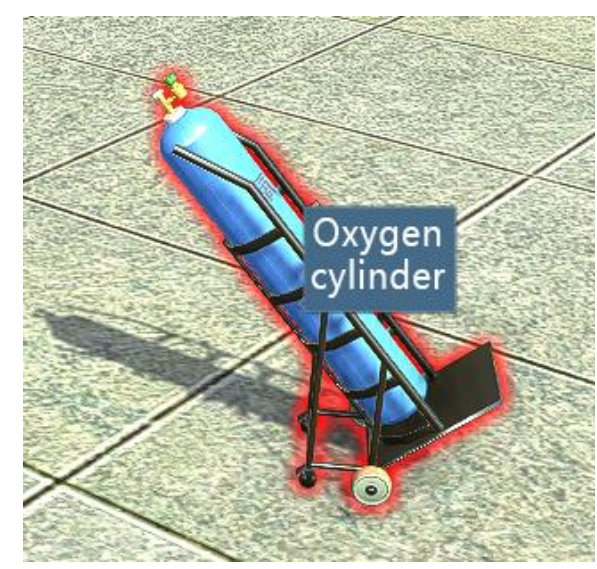

Fig. 3. Outline highlighting

\subsection{EBAT and self-built scenes}

The accident proportion of petrochemical direct operation is high and most of them are 
caused by human factors, so it's necessary to train operators by a large number of events.

EBAT refers that the operators are trained in various events and provided different feedback for different event operations in virtual environment, so related skills of trainers can be improved.This method is often incorporated into multimodal training programs, including teaching, demonstration, practice, and feedback, and EBAT is used to build the project's practices and feedback components[12]. Namely, EBAT creates connection between the training requirement (learning objective is rooted in a teamwork), training design (including trigger or key events provide students with the opportunity to demonstrate their mastery of the predefined goals), and observation and feedback (according to the students reaction events)[13].

However, direct operation has the characters of complexity, changeability and so on. The training contents in different situations are not the same, so a small number of training situations and events where operators can't train exhaustive and effective. In order to cope with various possible operation requirements and use the EBAT better in the direct operation training, the virtual simulation system needs to simulate enough operation events to train operators.

Therefore, we add the edit layer to accomplish the self-built scenes and self-edited courses. We build scenes according to 3D models and other data on the system bottom layer. Then we extract task characteristics in the operation process to divide the responsibilities of different operators. Next we describe the virtual operators, operation type and operation abstractly and store them with single operation step. Finally, we combine different operation steps in seriesparallel connection through "and", "or" logical relationship according to pre-edited operation process script, so different operation organization structure and training process can be constructed flexibly and the basic framework of self-edited courses is achieved. Scene building interface and course editing interface are shown as Figure 4, 5, 6.

After editing course, the participants can interact with the training environment.Clearly, operation management and motion control are key aspects of this process. Training participants must be allowed to make their own decisions, and in a behavior consistent with the regulation of simulation, and the operation managers must ensure that the correct type of opportunity in a controlled manner, in order to achieve the training goals[14].

From this framework, experts of direct operation can edit 3D simulation training courses with real operating processes and high risk points and expand training events without limit. The problems of insufficient training scenes and insufficient training events of EBAT can be solved, therefore EBAT can be better fused into the direct operation simulation training.

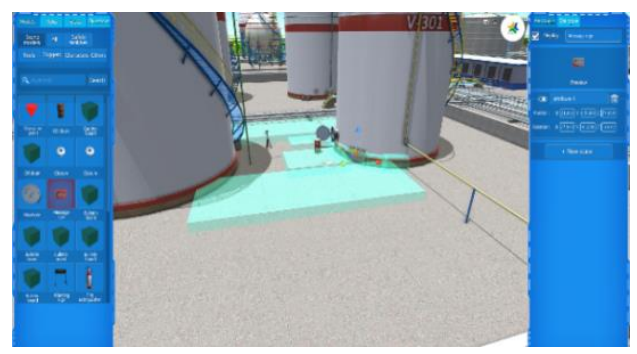

Fig. 4. Scene building

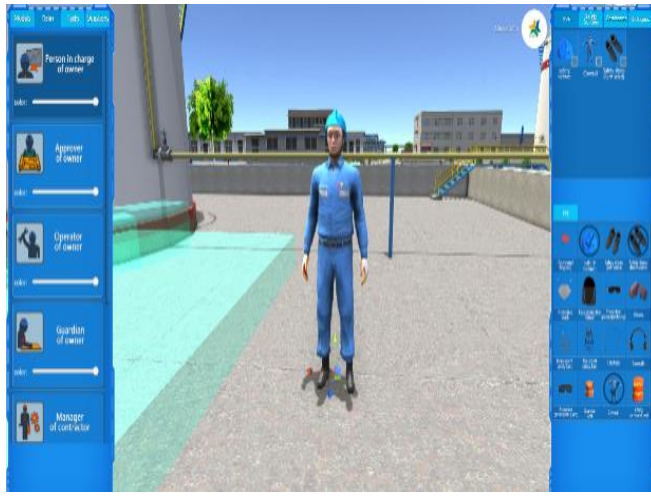

Fig. 5. Role setting

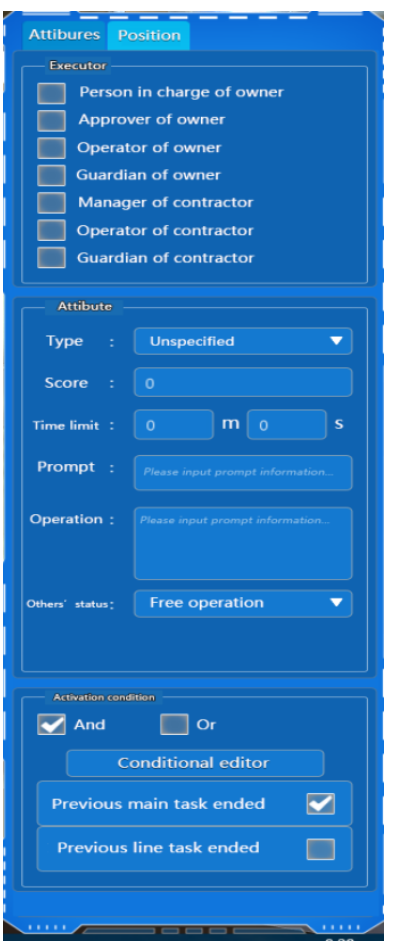

Fig. 6. Process setting

\subsection{Automatic examination and evaluation}

It is very important for an assessment section to score automatically in terms of participation and operation of the trainer. The corresponding score information is stored in the process in edit layer according to importance degree of the process. There are three kinds of training tasks: examination questions, inquiry and equipment operation. The role of the trainer 
is different from the training task. Each link is scored in real time, According to the selection and interaction of the trainers. Feedback is given to the trainer by displaying the correct operation in the window form for the error operation. The error options will be recorded, and the trainers can then practice the low score parts independently, while the other operators are controlled by the AI module.

The total score of all the tasks will be calculated after training, including validity of the examination problems, standard of the interaction and operation time in each procedure. Then the score will be converted to centesimal system.

\subsection{Multi-agent collaboration}

Virtual simulation training can be divided into three types: closed demonstration, guided operation and open operation training mode [11]. Closed demonstration requires that the system performs an operation demonstration with operating rules strictly. Guided operation training mode means that system tells the operator how to complete the operation. Open operation training mode means that the operator can do all operations for all functional units. Direct operation is carried out under a certain operation flow which can't be disrupted, therefore, the simulation training in this paper is designed mainly in guided operation training mode. Taking the correct operation process and the corresponding operation steps as the guide, the virtual simulation guides the trainer to perform $3 \mathrm{D}$ interaction in the virtual scene with some operation hints.

In order to improve the interactivity of the simulation system and effectiveness of training, we use multi-role distributed interactive simulation. The implementation of a direct operation requires the participation of seven types of roles. In order to simulate multi-role participation in the direct operation, we adopt the distributed interactive simulation technology to achieve the interaction among different roles in the scene.

To solve the problem that all the seven types of characters may not participate at the same time in training, we propose the roles automatic communication and interaction based on AI (Artificial Intelligence), that is to say, the system would automatically perform all tasks of the roles without training control through the method of AI, including communication with others and interaction with the scene, to insure that the operation process is gone on smoothly.

The operation of seven types of operators may be the same, which means that different roles may perform the same interaction with the scene at the same time. In this case, we apply selective communication solutions to make sure the logic is reasonable, that is to say, in the same scene, the 3D interaction of different clients can't be affected by others. The client's operations are executed and displayed locally to avoid affecting the interaction of other clients through network communication. In this way, each client has its own interaction logic and realtime communication with other clients at the same time .

\section{Experiments and analysis}

Based on the above methods, a simulation system for petrochemical workers is developed, which is divided into three layers with the thought of lamination, they are data layer, edit layer and training layer, as shown in Figure 7. The data layer at the bottom of the system mainly stores the 3D auxiliary resource model and other forms of data created by 3ds Max; The edit layer is the part that designers build scenarios and edit courses based on operation process; The training layer is the part that trainers execute virtual simulation training. Users with different permissions have corresponding operation. The system business logic is shown in Figure 8.

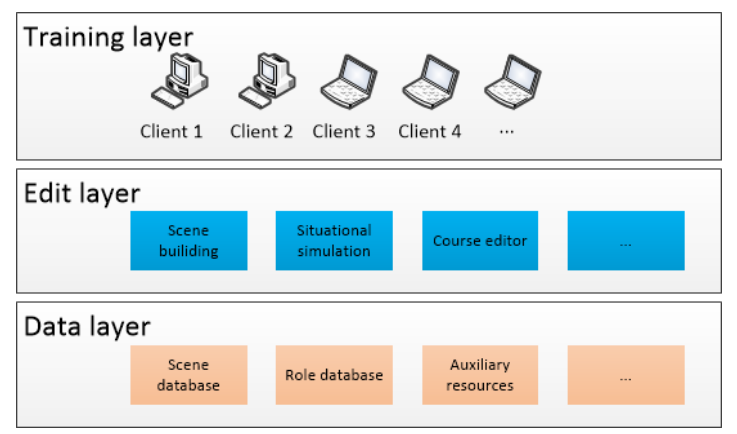

Fig. 7. The three layers of the simulation system 


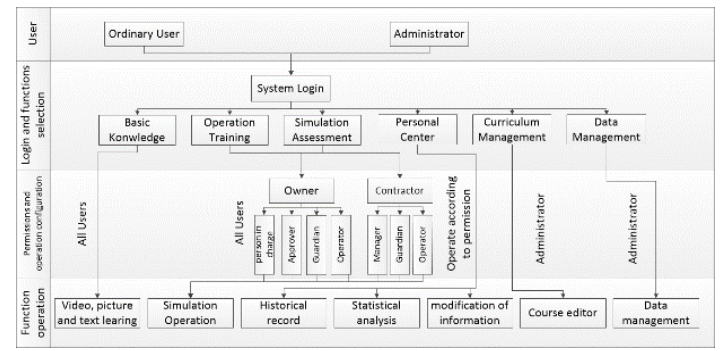

Fig. 8. The system business logic

The two-dimensional interactive interface of the system is design by UGUI in Unity3D. The system imports and exports 3D models by "AssetBundle". and then uploads them to FTP server. SQL Server stores Auxiliary 3D models, pictures and voices. FileZilla server interface is shown in Figure 9.

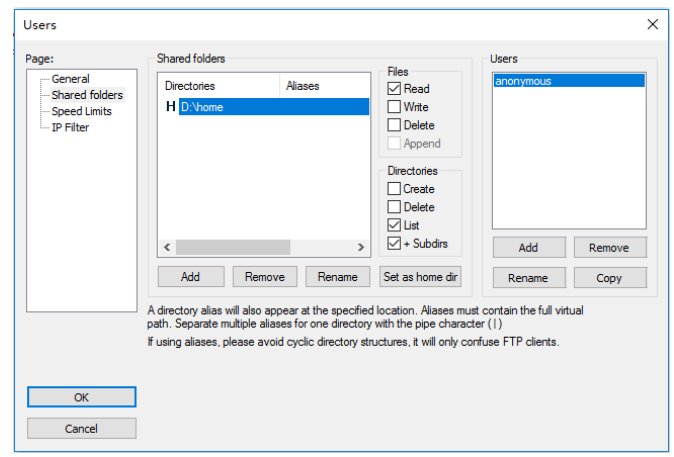

Fig. 9. FTP interface setting

In this system, virtual scenes are instead of real scenes, and virtual characters are instead of operation people. Virtual scenes is modeled using $3 \mathrm{ds}$ max by one to one ratio according to the real scenes of petrochemical plant and tank farms of a petrochemical enterprise, as shown in Figure 10(a). Virtual operation people are modeled according to height proportion of real people and they contain behavior information such as walking, running, jumping, squatting and others, as shown in Figure 10(b).

Abrupt change of weather may occur during the direct operation. Weather such as rain and snow effect is simulated through Particle System, as shown in Figure 10(c). The system determines whether the model is in correct position by Trigger and distance limit. Outline highlight is enhanced by the rim light. There will be distance restriction from each other between two models, and the line will be shown between two models in the three-dimensional scene in order to show the distance clearly, as shown in 10(d). Each step contains corresponding fractional information, therefore users can get scoring evaluation through the score hundred differentiation. Eventually the system is published on the $\mathrm{PC}$ with $\mathrm{C} / \mathrm{S}$ architecture.

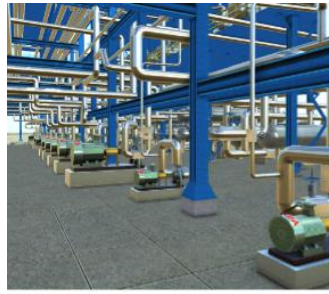

(a)Virtual Scene

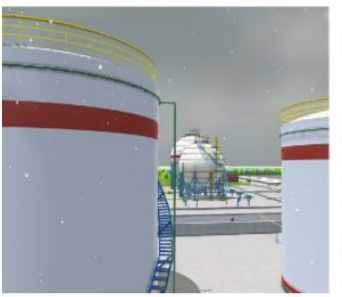

(c)Snowy Weather Simulation

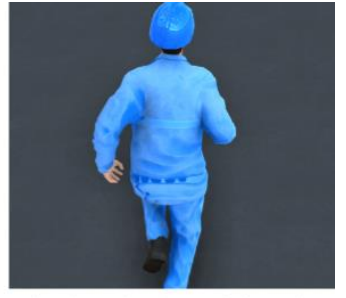

(b) The Behaviour of the Role

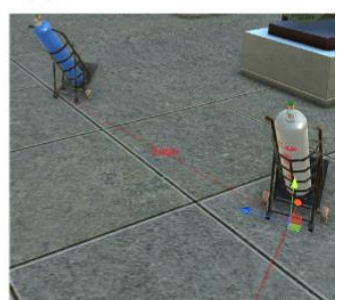

(d)Drawing line by OpenGL

Fig. 10. Display of system performance 
We take the role of the job manager as an example to describe the training process, as shown in Figure 11. The operator conducts the simulation training according to the expert edited work flow. For each task, the system automatically scores and feedbacks errors according to the correctness of the operator's answer, the accuracy of the interaction, and the time cost of the operation.After finish all training, the system displays final score and evaluation report.The operator can independently train the assignments with low scores, while other roles are controlled by AI module.

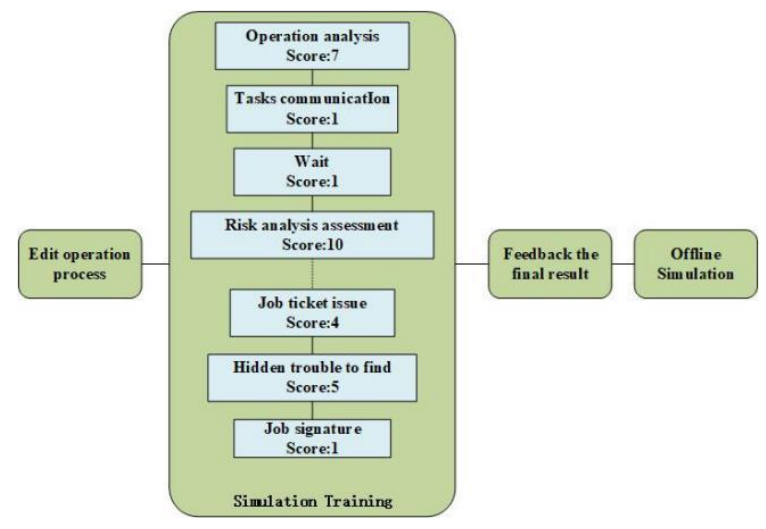

Fig. 11. Training Process

\section{Conclusions}

The simulation of the direct operation developed by virtual reality technology can reproduce reality scene and allow relevant people to interact with virtual scene, and it's not affected by time and place. It is a new method to reduce accidents in direct operation.

The virtual reality simulation training is applied to the virtual simulation of petrochemical direct operation in this paper, and the direct operation simulation training system has been designed and implemented by Untiy3D.

The system solves the problems of complex scenes and pipelines of petrochemical plant, and builds a three-dimensional scene of a petrochemical plant realistically using a new 3D modeling method based on large scale topographical map and chemical process. It contains rich build-in 3D model resource and 2D basic knowledge resources, which allows users to build their own scenarios and edits direct operation courses. Training courses can be expanded without limit, the expansibility and applicability of the system are improved, so EBAT can be better applied to the direct operation simulation training. Roles can be better fused into the simulation scene using multi-role multi-modal interaction. The simulation of petrochemical direct operation is great significant to reducing accidents in reality operation.

Our next work is to transfer the simulation system to the mobile terminal with the portability of Unity3D, so that the trainers can use the system for training anytime and anywhere.

Acknowledgments. This paper is supported by the Key Research\&Development Plan Project of Shandong Province (No.2017GGX10127, 2017GSF18110, 2018GGX101029) and Natural Science Foundation of Shandong Province(No.ZR2014FM015).

\section{References}

[1] Zhang Linxuan, Xin Xianjie, Cui Bing, Ni Lebo, Liu Jun, Xiao Tianyuan. Research on Automotive Product Design Oriented Virtual Reality Service Platform [J]. Journal of systems simulation. 2014, 26(10): 2407-2411.

[2] He Gaoqi, Yang Kailin, Jin Wei, Chen Qi, Li Haijie, Pan Zhigeng. Interactive Illustration Technologies for Energy Stations Using Unity3D [J]. Journal of systems simulation.2016, 28(10): 2626-2631.

[3] Wu Dandan. X3D-Based Research and Realization of CNG Simulation Training System [D]. Southwest Petroleum University.2011.

[4] Yao Pengfei, Chen Zhengming, Tong Jing, Qian Lina. Virtual Simulation System of Cutter Suction Dredger Based on Unity3D [J]. Journal of systems simulation.2016, 28(9): 2069-2075.

[5] Yue Yanyan. Research on Virtual Simulation Training System of Small-scale Workover Based on Multi-user Collaboration [D]. Northeast Petroleum University. 2016.

[6] Fowlkes J E, Dwyer D J, Oser R L, Salas E. Event-based Approach to Training (EBAT) [J]. International Journal of Aviation Psychology (S1532-7108), 1998, 8(3): 209-221.

[7] Wang Jian, Wan Hongwei. Research on Team Simulation Training Functional Framework Based on HLA [J]. Journal of systems simulation. 2010, 22(5):1164-1168. 
[8] Wang Jian, Wan Hongwei. Scenario Generation Approach for Team Simulation Training Based on Bayesian Network [J]. Journal of systems simulation. 2011, 23(2):339-344;

[9] Xu Gangyan, Wang Jian, Wang Hongwei, Liu Benxu, Li Jingjing. Scenario Generation and Scheduling of National Economy Mobilization Simulation and Training System [J]. Journal of systems simulation. 2012, 24(7): 1485-1490.

[10]Hou Lihua, Yang Fangting, Ji Liangxiong, Zhao Longjun, Chen Jirong, Han Jun. The Design and Development of Simulation System for Chemical Process [J]. Journal of systems simulation. 2003, 15(9): 1307-1310.

[11]Liu Xianmei, Hao Aimin. Virtual Simulation for Training of Oil-field Safety Task Operation [J]. Journal of systems simulation. 2006, 18(11): 3082-3087.

[12]Fowlkes JE, Lane NE, Salas E, Franz T, Oser R. Improving the measurement of team performance: the TARGETs methodology. Mil Psychol. 1994;6(1):47-61.

[13]Nguyen N, Watson W D, Dominguez E. An Event-Based Approach to Design a Teamwork Training Scenario and Assessment Tool in Surgery[J]. Journal of Surgical Education, 2016, 73(2):197207.

[14]Oser R L, Gualtieri J W, Cannonbowers J A. Enhancing Training Systems Design: Implementing an Event-Based Approach[C]// 1998:1309-1313. 